\title{
GISTS MÚLTIPLOS EM NEUROFIBROMATOSE TIPO 1: DIAGNÓSTICO INCIDENTAL EM PACIENTE COM ABDOME AGUDO
}

\author{
Multiple GISTs in neurofibromatosis type 1: incidental diagnosis in a patient with \\ acute abdomen
}

\author{
Tomaz de Jesus Maria GREZZANA-FILHO, Taís Burmann de MENDONÇA, Liane GOLBSPAN, \\ Cleber Rosito Pinto KRUEL, Aljamir Duarte CHEDID, Cleber Dario Pinto KRUEL
}

ABCDDV/643

Grezzana-Filho RJM, Mendonça TB, Golbspan L, Kruel CRP, Chedid AD, Kruel CDP. Gists múltiplos em neurofibromatose tipo 1: diagnóstico incidental em paciente com abdome agudo. ABCD Arq Bras Cir Dig 2009;22(1):65-8

RESUMO - Introdução - Tem sido descrito na literatura incidência aumentada de tumores estromais gastrointestinais (GISTs) em portadores de neurofibromatose tipo 1. Estes tumores tipicamente ocorrem no intestino delgado e, frequentemente, são múltiplos. Relato do caso - Diagnóstico incidental de GIST em um paciente portador de neurofibromatose tipo $1 \mathrm{com}$ abdome agudo. No trans-operatório foi identificada apendicite retrocecal perfurada e massa neoplásica no jejuno proximal. A referida lesão ocupava aproximadamente $70 \%$ da circunferência do órgão e não apresentava invasão de estruturas adjacentes. Além disso, observaram-se dezenas de pequenos nódulos disseminados por toda a extensão do jejuno e íleo. O anatomopatológico revelou neoplasia compatível com GIST, com grau moderado de atipias, baixo índice mitótico $(<5 / 50)$ e ausência de necrose. Na análise imunoistoquímica, as células neoplásicas foram positivas para CD-34 e CD-117 (c-KIT), e negativas para a desmina. Depois da operação, o uso do quimioterápico Imatinib foi indicado. Após período de acompanhamento de 12 meses, o paciente não demonstrou sinais de recidiva. Conclusão - A ocorrência de GISTs deve ser sempre lembrada na avaliação de pacientes com massas abdominais e neurofibromatose tipo 1, principalmente em intestino delgado. Muitas vezes o comportamento da neoplasia é favorável e o uso de Imatinib deve ser individualizado. DESCRITORES - Neurofibromatose. Tumor estromal gastrointestinal. Abdome agudo.

\section{INTRODUÇÃO}

Os tumores do estroma gastrointestinal (GISTs) ocorrem predominantemente de forma esporádica e representam cerca de $1 \%$ de todas as neoplasias do trato gastrointestinal ${ }^{12}$. As evidências atuais apontam que a origem dos GISTs tem relação direta com as células intersticiais de Cajal, as quais fazem parte do plexo mioentérico do trato digestivo e são responsáveis pelo controle da motilidade intestinal'. Em geral os GISTs são neoplasias bem delimitadas que se projetam exofiticamente em direção à luz do órgão envolvido. Podem provocar sintomas obstrutivos e sangramento agudo ou crônico. Com menor frequência eles se expandem através da serosa e seu diâmetro pode atingir dezenas de centímetros ${ }^{2}$.

A neurofibromatose tipo 1 (NF-1) ou doença de Von Recklinghausen é uma das desordens genéticas mais comuns e afeta cerca de 1 em cada 3.500 indivíduos $^{3}$. Ela engloba um amplo espectro de manifestações patológicas que aparecem em diversas partes do corpo, com envolvimento dos tecidos cutâneo, ósseo, nervoso e de partes

Trabalho realizado no Hospital de Clínicas de Porto Alegre - Departamento de Cirurgia, Porto Alegre, RS, Brasil. Endereço para correspondência:

Tomaz de Jesus Maria Grezzana Filho, e-mail: tomgrez@uol.com.br moles. Os achados mais comuns são manchas cutâneas do tipo "café-com-leite" é o achado de neurofibromas de pele disseminados pela superfície corporal. Os indivíduos portadores apresentam risco aumentado de desenvolver neoplasias malignas em geral, incluindo tumores carcinóides, feocromocitoma e GISTs $^{14}$. Em uma série de necropsias em pacientes com neurofibromatose tipo 1, foi detectada a presença de GISTs incidentais em um terço dos indivíduos ${ }^{5}$. No entanto, a verdadeira incidência dos GISTs e a possibilidade destes tumores se tornarem clinicamente significantes não é conhecida.

O presente artigo relata um caso incomum de múltiplos GISTs associados à neurofibromatose tipo 1 que foram diagnosticados incidentalmente em paciente apresentando quadro de abdome agudo.

\section{RELATO DO CASO}

Homem de 61 anos foi admitido na emergência do Hospital de Clínicas de Porto Alegre, Porto Alegre, RS, Brasil apresentando há uma semana dor intensa na região lombar direita, febre, inapetência e vômitos biliares. À ectoscopia, apresentava neurofibromas disseminados por toda a superfície corporal. No exame abdominal foram constatados evidentes sinais de irritação peritoneal na fossa ilíaca direita. Os exames laboratoriais 
revelaram anemia microcítica e leucocitose com desvio à esquerda. Ultrassonografia do abdome demonstrou lesão expansiva no mesogástrio de natureza indeterminada. No entanto, tomografia de abdome mostrou uma lesão sólida medindo 4,8 x 4,4 x 4,6 cm à esquerda da aorta, com calcificações periféricas (Figura 1). Outra lesão de limites imprecisos e infiltrativa medindo cerca de $5,1 \mathrm{x}$ $4,2 \times 4,9 \mathrm{~cm}$ foi detectada no quadrante inferior direito podendo ser de origem neoplásica ou um plastrão inflamatório.

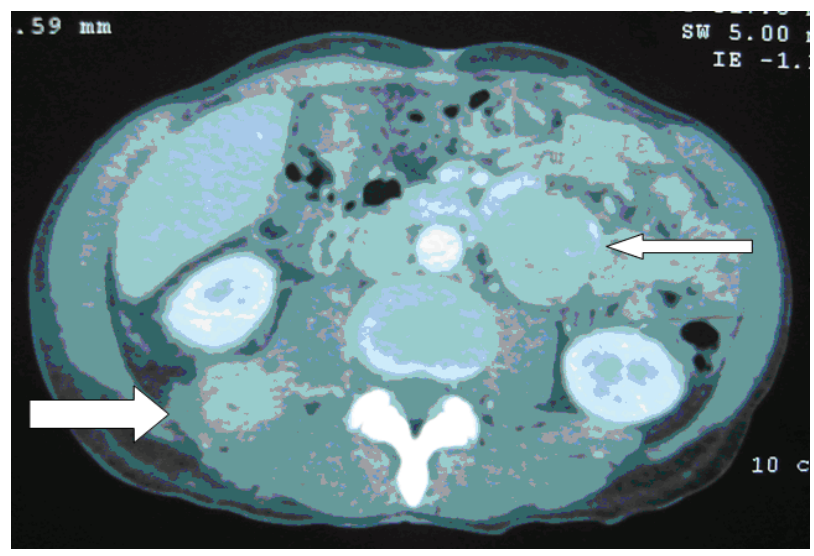

FIGURA 1 - Aspecto tomográfico de lesão expansiva no mesogástrio (seta estreita) e lesão de limites imprecisos na fossa ilíaca direita (seta larga)

O paciente foi submetido a laparotomia exploradora. No trans-operatório foi identificada apendicite retrocecal perfurada envolta em grande plastrão inflamatório, que foi adequadamente tratada. Uma massa neoplásica no jejuno proximal, próxima ao ângulo duodeno-jejunal foi identificada e ressecada. O trânsito intestinal foi reconstruído através de enteroentero anastomose terminal. A referida lesão ocupava aproximadamente $70 \%$ da circunferência do órgão e não apresentava invasão de estruturas adjacentes. Além disso, observaram-se dezenas de pequenos nódulos disseminados por toda a extensão do jejuno e íleo. Foi realizada biópsia excisional em um deles.

$\mathrm{Na}$ macroscopia foi descrita lesão vegetante e ulcerada, medindo 5,0 x 4,3 cm. O exame anatomopatológico revelou neoplasia de células fusiformes do jejuno, com invasão do mesentério, compatível com GIST. Nos critérios de estratificação de risco, a neoplasia apresentava grau moderado de atipias, baixo índice mitótico $(<5 / 50)$ e ausência de necrose (Tabela $1)^{4}$. A biópsia do pequeno nódulo mostrou os mesmos resultados. Os linfonodos mesentéricos foram negativos para neoplasia.

$\mathrm{Na}$ análise imunoistoquímica, as células neoplásicas foram positivas para CD-34, CD-117 (c-KIT) e negativas para a desmina, perfil compatível com GIST (Figura 2).

O paciente evoluiu sem complicações e recebeu alta no sétimo dia do pós-operatório.
TABELA 1 - Parâmetros para definir comportamento dos GISTs

\begin{tabular}{lll}
\hline $\begin{array}{l}\text { Risco de comportamento } \\
\text { agressivo }\end{array}$ & Tamanho do Tumor & $\begin{array}{l}\text { Número de mitoses por } \\
\text { campo }\end{array}$ \\
\hline Muito baixo & $<2 \mathrm{~cm}$ & $<5 / 50 \mathrm{HPF}$ \\
Baixo & $2-5 \mathrm{~cm}$ & $<5 / 50 \mathrm{HPF}$ \\
Intermediário & $<5 \mathrm{~cm}$ & $6-10 / 50 \mathrm{HPF}$ \\
& $5-10 \mathrm{~cm}$ & $<5 / 50 \mathrm{HPF}$ \\
Alto & $>10 \mathrm{~cm}$ & Qualquer número \\
& Qualquer tamanho & $>10 / 50 \mathrm{HPF}$ \\
\hline
\end{tabular}

HPF $=$ High - Power Field

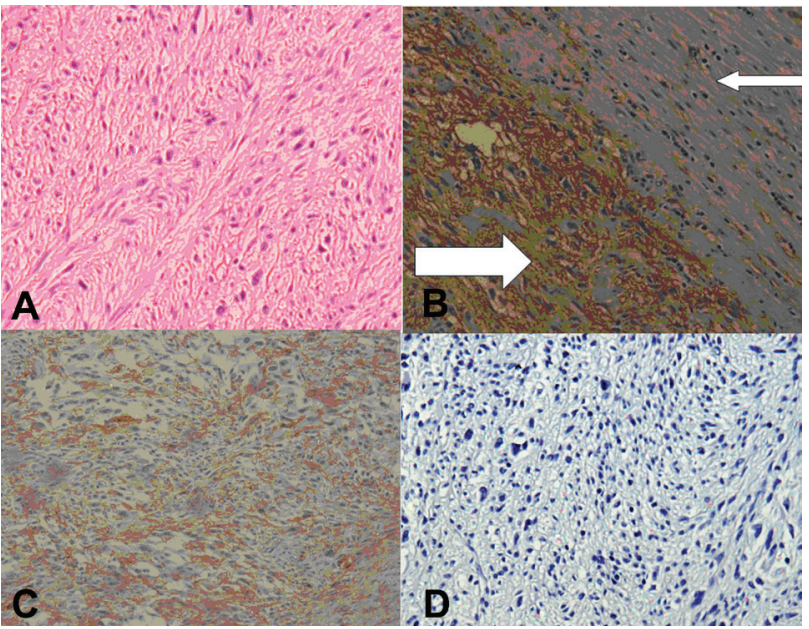

FIGURA 2 - GIST jejunal: A) aparência típica de tumor de células fusiformes, atipias moderadas e baixo índice mitótico ( $<5 / 50 \mathrm{HPF}$ - HE, 200x); B) Neoplasia positiva para CD-34 (seta larga) com camada muscular negativa para CD-34 (seta estreita), 200x; C) neoplasia positiva para CD-11, 200x; D) neoplasia negativa para desmina, 200x.

\section{DISCUSSÃO}

Os GISTs são neoplasias muitas vezes assintomáticas e frequentemente diagnosticadas de forma ocasional. Estes tumores costumam ter comportamento imprevisível, sendo difícil predizer sua evolução. Contudo, consenso foi publicado em 2002 a fim de estimar o risco de comportamento agressivo dos GISTs. Essa recomendação leva em conta o tamanho tumoral e a contagem mitótica ${ }^{4}$. Além disso, fatores como a localização intestinal e a presença de necrose possivelmente se relacionam também a um comportamento agressivo ${ }^{9}$. Devido a desordens genéticas provocadas pela neurofibromatose tipo 1 , os pacientes portadores desta síndrome têm chance 45 vezes maior de desenvolver um GIST $^{8}$.

O presente relato está em conformidade com outras publicações, que teem demonstrado que a associação de GIST e neurofibromatose tipo 1 não é casual ${ }^{1,7,10,14,15}$. Em particular, a faixa etária acometida pelos GISTs na neurofibromatose tipo 1 é menor do que em casos isolado5. Tem-se observado também que a associação de GIST e neurofibromatose tipo 1 aparece com maior frequência no jejuno e no íleo, outra característica observada no caso relatado, enquanto que os casos esporádicos são observados 
com maior frequência no estômago (60\%)9. Em uma série de 15 pacientes com GIST e neurofibromatose tipo 1, todos apresentavam lesões no intestino delgado e em outra série de 45 pacientes, apenas um indivíduo apresentava lesões no estômago ${ }^{5}$. Outra característica peculiar é que os GISTs associados à neurofibromatose tipo 1 tendem a ser múltiplos e multicêntricos ${ }^{10}$. Em uma série de 288 pacientes com GIST, apenas cinco indivíduos apresentavam lesões multicêntricas e todos eram portadores de neurofibromatose tipo 119. Em outra série de 15 casos de GISTs associados a essa doença, todos indivíduos apresentavam lesões múltiplas e um deles tinha mais de 100 lesões macroscópicas ${ }^{6}$.

Morfologicamente, os casos associados à neurofibromatose tipo 1 e esporádicos são indistinguíveis. Atualmente, a imunoistoquímica permite o diagnóstico diferencial entre os GISTs e os leiomiomas com maior confiabilidade, já que são neoplasias muito semelhantes e de prognósticos muito diferentes ${ }^{12}$. No presente relato, a ausência de positividade para o marcador desmina descartou a origem muscular da neoplasia. A maioria dos GISTs expressa os receptores CD-117, CD-34 e PDGFRA (Platelet Derived Grow Factor Receptor Alfa) $)^{2,8,12,13}$. No presente relato, a positividade para CD-34 e c-KIT demonstrou perfil compatível com GIST. Entretanto, acredita-se que os GISTs associados à neurofibromatose tipo 1 surjam de uma rota alternativa, já que a positividade para mutações c-KIT e PDGFRA muitas vezes não é encontrada ${ }^{7,9}$, diferente dos GISTs esporádicos que apresentam tais mutações.

A maioria dos GISTs em indivíduos com neurofibromatose tipo 1 tem baixa atividade mitótica e morfologia fusiforme, características associadas a um melhor prognóstico ${ }^{8}$. Isso reflete o comportamento indolente dos tumores que ocorrem associados com a neurofibromatose tipo 1. Já os casos esporádicos teem desfecho clínico menos favorável pois costumam apresentar maior atividade mitótica e a morfologia epitelióide, relacionada a mau prognóstico também é mais frequente ${ }^{11}$.

O tratamento de escolha para esses tumores é a ressecção total. Não há necessidade de margens cirúrgicas extensas e não se realiza dissecção linfonodal rotineira, uma vez que as metástases ganglionares são raras. Os principais sítios metastáticos são o fígado e o peritônio. Nesses casos, a probabilidade de tratamento curativo é muito baixa.

O quimioterápico Imatinib atua como inibidor seletivo da tirosina kinase, do receptor PDGFRA e do receptor cKIT. A terapia com Imatinib tem boa tolerabilidade oral e apresenta efeitos positivos principalmente nos casos de recidiva e metástases ${ }^{11,12}$. No presente relato, o GIST ressecado mediu 5,0 x 4,3 cm nos maiores eixos e tinha baixo índice mitótico, características compatíveis com prognóstico favorável. Entretanto, já que apresentava perfil imunoistoquímico com sensibilidade ao Imatinib e potencial de crescimento das lesões disseminadas pelo abdome, o uso do quimioterápico foi indicado. Após período de acompanhamento de 12 meses, o paciente não demonstrou sinais de recidiva.

\section{CONCLUSÃO}

A ocorrência de GISTs deve ser sempre lembrada na avaliação de pacientes com massas abdominais e neurofibromatose tipo 1. Estes tumores tipicamente ocorrem no intestino delgado e frequentemente são múltiplos. Muitas vezes o comportamento da neoplasia é favorável e o uso de Imatinib deve ser individualizado.

Grezzana-Filho RJM, Mendonça TB, Golbspan L, Kruel CRP, Chedid AD, Kruel CDP. Multiple GISTs in neurofibromatosis type 1: incidental diagnosis in a patient with acute abdomen. ABCD Arq Bras Cir Dig 2009;22(1):65-8

ABSTRACT - Background - The literature described an increased incidence of gastrointestinal stromal tumors (GISTs) in patients with neurofibromatosis type 1 . These tumors typically occur in the small intestine, and frequently are multiple. Often the behavior of the tumor in this association is more favorable than in sporadic cases. Case report - Incidental diagnosis of GIST was done in a patient with neurofibromatosis type 1 during treatment for acute abdomen. Trans-operatively was identified a retrocecal perforated appendicitis and a neoplastic mass in the proximal jejunum. The lesion occupied approximately $70 \%$ of the circumference of the organ and had no invasion of adjacent structures. Moreover, there were dozens of small nodules scattered throughout the length of the jejunum and ileum. The pathology revealed malignancy consistent with GIST, with moderate degree of atypia, low mitotic index $(<5 / 50)$ and absence of necrosis. In immunohistochemical analysis, the neoplastic cells were positive for CD-34 and CD-117 (c-KIT), and negative for desmin. After surgery, the use of Imatinib chemotherapy was indicated. After a follow-up period of 12 months, the patient showed no signs of recurrence. Conclusion - GISTs should be considered in the presence of abdominal mass and neurofibromatosis type 1, affecting mainly small bowel. Sometimes Imatinib can be administered with good results in the control of the disease.

HEADINGS - Neurofibromatosis. Gastrointestinal stromal tumors. Acute abdomen.

\section{REFERÊNCIAS}

1. Cheng SP, Huang MJ, Yang TL, et al. Neurofibromatosis with gastrointestinal stromal tumors: insights into the association. Dig Dis Sci. 2004;49:1165-1169.

2. Fenaglio-Preiser CM - Gatrointestinal Pathology. 1 ed., New York, Raven Press, 1989. pp 543-585.

3. Ferner RE, Huson SM, Thomas N, Moss C, Willshaw H, Evans DG et al.. Guidelines for the diagnosis and management of individuals with neurofibromatosis 1. J Med Genet 44:81-8, 2007.

4. Fletcher CDM, Berman JJ, Corless C, Gorstein F, Lasota J, Longley BJ, Miettinem M, O'lerary T, Remotti H, Rubin BP, Shmookler B, Sobin LH, Weiss SW Diagnosis of Gastrointetinal Stromal Tumors: A consensus approach. Human Pathology; 33(5): 459-465, may 2002.
5. Fuller CE,Williams GT. Gastrointestinal manifestations of type 1 neurofibromatosis (von Recklinghausen's disease). Histopathology. 1991;19:111

6. Johanna Andersson, Harri Sihto, Jeanne M. Meis-Kindblom, Heikki Joensuu, Nina Nupponen, Lars-Gunnar Kindblom. NF1-Associated Gastrointestinal Stromal Tumors Have Unique Clinical, Phenotypic, and Genotypic Characteristics. Am J Surg Pathol Volume 29, Number 9, September 2005

7. Kinoshita K, Hirota S, Isozaki K, et al. Absence of c-kit gene mutations in gastrointestinal stromal tumours from neurofibromatosis type 1 patients. J Pathol. 2004; 202:80-85. 
8. Miettinen M, Fetsch JF, Sobin LH and Lasota J: Gastrintestinal stromal tumors in patients with neurofibromtosis 1. A Clinicopathologic and Molecular Genetic Study of 45 cases. Am J Surg Pathol (2006) 30: 90-96.

9. Miettinen M, Lasota J. Gastrointestinal stromal tumors: definition, clinical, histological, immunohistochemical, and molecular genetic features and differential diagnosis. Virchows Arch. 2001;438:1-12

10. Min KW, Balaton AJ. Small intestinal stromal tumors with skeinoid fibers in neurofibromatosis: report of four cases with ultrastructural study of skeinoid fibers from paraffin blocks. Ultrastruct Pathol. 1993;17:307- 314.

11. 11 - Nilsson B, Bumming P,Meis-Kindblom JM, et al. Gastrointestinal stromal tumors. The incidence, prevalence, clinical course and prognostication in the pre-imatinib era: a population-based study in western Sweden. Cancer. 2005;103:821-829

12. 12 - Silva Fe, Ascoly MH, Scofano V, Arakaki JR N, Reis O, Sá MAGS Tumores Estromais Gastrointestinais- GIST: Relato de um Caso. Rev Bras Coloproct, 2004; 24(2): 159-164.
13. 13 - Takazawa Y, Sakurai S, Sakuma Y, et al. Gastrointestinal stromal tumor of neurofibromatosis type 1 (von Recklinghausen's disease). Am J Surg Pathol. 2005;29:755-763.

14. 14 - Yantiss RK, Rosenberg AE, Sarran L, et al. Multiple gastrointestinal stromal tumors in type I neurofibromatosis: a pathologic and molecular study. Mod Pathol. 2005; 18:475-484.

15. 15 - Zoller ME, Rembeck B, Oden A, et al. Malignant and benign tumors in patients with neurofibromatosis type 1 in a defined Swedish population. Cancer. 1997;79:2125-213

Fonte de financiamento: não há Conflito de interesse: não há Recebido para publicação: 05/07/2008 Aceito para publicação: 13/10/2008 